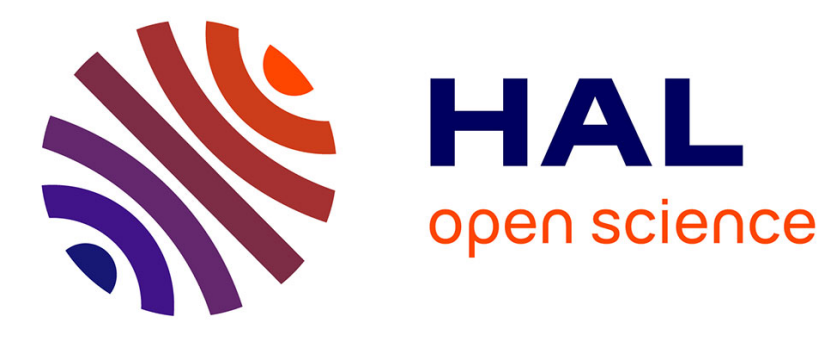

\title{
New asymptotic properties for the robust ANMF
}

Gordana Draskovic, Frédéric Pascal, Arnaud Breloy, Jean-Yves Tourneret

\section{To cite this version:}

Gordana Draskovic, Frédéric Pascal, Arnaud Breloy, Jean-Yves Tourneret. New asymptotic properties for the robust ANMF. The 42nd IEEE International Conference on Acoustics, Speech and Signal Processing (ICASSP 2017), Mar 2017, New Orleans, United States. 10.1109/ICASSP.2017.7952793 . hal-01816123

\section{HAL Id: hal-01816123 \\ https://hal.science/hal-01816123}

Submitted on 9 Apr 2020

HAL is a multi-disciplinary open access archive for the deposit and dissemination of scientific research documents, whether they are published or not. The documents may come from teaching and research institutions in France or abroad, or from public or private research centers.
L'archive ouverte pluridisciplinaire HAL, est destinée au dépôt et à la diffusion de documents scientifiques de niveau recherche, publiés ou non, émanant des établissements d'enseignement et de recherche français ou étrangers, des laboratoires publics ou privés. 


\title{
NEW ASYMPTOTIC PROPERTIES FOR THE ROBUST ANMF
}

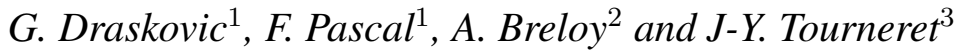 \\ ${ }^{1}$ L2S, CentraleSupélec, 3 rue Juliot Curie, 91190 Gif-surYvette, France \\ ${ }^{2}$ LEME, University Paris Ouest, 92410 Ville d'Avray, France \\ 3 IRIT, ENSEEIHT, 2 rue Charles Camichel, 31071 Toulouse, France
}

\begin{abstract}
The purpose of this paper is to derive new asymptotic properties of the robust adaptive normalized matched filter (ANMF). More precisely, the ANMF built with Tyler estimator (TyEANMF) is analyzed under the framework of complex elliptically symmetric (CES) distributions. We show that the distribution of TyE-ANMF can be accurately approximated by the well-known distribution of the ANMF built with the sample covariance matrix (SCM-ANMF) under the Gaussian assumption. To that end, the asymptotic properties of the difference between both ANMF detectors are derived. By comparison with the state of the art, the asymptotic properties of the TyE-ANMF are shown to be better approximated by the SCM-ANMF rather than using the NMF (test built with the true $\mathrm{CM}$ ). Some Monte-Carlo simulations support that claim and demonstrate the interest of this theoretical result.
\end{abstract}

Index Terms- Adaptive Normalized Match Filter, Complex Elliptically Symmetric distributions, Sample Covariance Matrix, Tyler estimator, robust estimation

\section{INTRODUCTION}

Detection theory has received a considerable attention in statistical signal processing. In particular, some detection problems that have been studied for radar processing can be found in [1-6]. In practice, the noise parameters are unknown and have to be estimated. These parameters include the noise covariance matrix $(\mathrm{CM})$ which is an important quantity since the performance of the main adaptive detectors strongly relies on its estimation accuracy. For instance, this is the case for the adaptive normalized matched filter (ANMF) [1]. In recent years there has been an increasing interest for deriving new $\mathrm{CM}$ estimators. Under the Gaussian assumption, the classical covariance estimator is the well-known sample covariance matrix (SCM) that is known to be distributed according to a Wishart distribution [7]. Since the SCM is a maximum likelihood estimator (MLE), it has nice asymptotic properties. However, it also suffers from major drawbacks. For instance, when the distribution of the training data is non-Gaussian, or when the data are corrupted by some outliers, the performance of the SCM generally degrades significantly.
Robust estimation theory offers an alternative to the SCM thanks to so-called $M$-estimators, that have been studied in the seminal works of Huber [8] and Maronna [9] in the real case. This has been recently extended to the complex case, which is sometimes more useful for signal processing applications, in [10]. Many recent works related to robust estimation have been conducted within the framework of complex elliptically symmetric (CES) distributions that encompass a large number of well-known distributions [11]. These $M$-estimators, especially Tyler estimator, also referred to as fixed-point (FP) estimator and denoted as $\widehat{\mathbf{M}}_{F P}[12,13]$, provide very good results when they are used in place of the true noise covariance matrix in many radar detectors. Unfortunately, the statistical analysis of the resulting adaptive detectors is difficult, mainly because of the non explicit form of these estimators. The asymptotic distribution of the ANMF built with $M$-estimators has been derived in [14]. The objective of this work is to better characterize the asymptotic distribution of the ANMF test built with Tyler estimator (TyE$\mathrm{SCM}$ ). To that end, we caracterize the asymptotic variance of the difference between the SCM-ANMF (ANMF based on the SCM) and the TyE-ANMF. Since the SCM-ANMF has well-known properties in the Gaussian context, an important consequence of the proposed result is to theoretically justify the use of the TyE-ANMF. More specifically, we will show that the TyE-ANMF has the same statistical properties as the SCM-ANMF, with the advantage of being more robust to the presence of outliers.

This paper is organized as follows. Section II introduces some background about CES and Wishart distributions, Tyler estimator and the ANMF. Section III presents the main contribution of the paper with discussions. Section IV shows Monte Carlo simulations validating the theoretical analysis. Finally, some conclusions are drawn in Section V.

Notations. Vectors (resp. matrices) are denoted by boldfaced lowercase letters (resp. uppercase letters). ${ }^{T}$ and ${ }^{H}$ are the transpose and Hermitian operators. $N$ and $\mathbb{C} N$ denote the real and complex normal distributions. $\sim$ means "distributed as", $\stackrel{d}{\rightarrow}$ denotes convergence in distribution and $\otimes$ denotes the Kronecker product. vec is the operator which transforms a matrix $m \times n$ into a vector of length $m n$, concatenating its 
$n$ columns into a single column. Moreover, $\mathbf{I}$ is the identity matrix, $\mathbf{0}$ the vector (or matrix) of zeros with appropriate dimension and $\mathbf{K}$ is the commutation matrix which transforms $\operatorname{vec}(\mathbf{A})$ into $\operatorname{vec}\left(\mathbf{A}^{T}\right)$.

\section{BACKGROUND}

\subsection{CES Distributions}

Let $\mathbf{z}$ be an $m$-dimensional complex circular random vector. This vector has a CES distribution if its probability density function (p.d.f.) can be written as

$$
h_{\mathbf{z}}(\mathbf{z})=|\mathbf{M}|^{-1} g_{\mathbf{z}}\left((\mathbf{z}-\boldsymbol{\mu})^{H} \mathbf{M}^{-1}(\mathbf{z}-\boldsymbol{\mu})\right)
$$

where $h_{\mathbf{z}}:[0, \infty) \rightarrow[0, \infty)$ is any function such that $(1)$ defines a PDF, $\boldsymbol{\mu}$ is the statistical mean and $\mathbf{M}$ is a scatter matrix. The matrix $\mathbf{M}$ reflects the structure of the covariance matrix of $\mathbf{z}$, i.e., the covariance matrix is equal to $\mathbf{M}$ up to a scale factor. This CES distribution will be denoted by $\mathbb{C} E S_{m}\left(\boldsymbol{\mu}, \mathbf{M}, g_{\mathbf{z}}\right)$.

Note that the Gaussian distribution is a particular case of CES distributions for which $g_{\mathbf{x}}(x) \propto e^{-x^{2}}$, that will be denoted $\mathbf{x} \sim \mathbb{C} N_{m}(\boldsymbol{\mu}, \mathbf{M})$. In this paper, we will assume that $\boldsymbol{\mu}=\mathbf{0}$. Without loss of generality, the scatter matrix will be equal to the covariance matrix when the latter exists. However, when the data distribution has a none finite second-order moment, then we will simply consider the scatter matrix estimator.

\subsection{Tyler estimator}

Let $\left(\mathbf{z}_{1}, \ldots, \mathbf{z}_{N}\right)$ be an $N$-sample of $m$-dimensional complex independent vectors with $\mathbf{z}_{k} \sim \mathbb{C} E S_{m}\left(\boldsymbol{\mu}, \mathbf{M}, g_{\mathbf{z}}\right)$. An $M$ estimator, denoted by $\widehat{\mathbf{M}}$, is defined by the solution of the following $M$-estimating equation

$$
\mathbf{M}=\frac{1}{N} \sum_{k=1}^{N} \varphi\left(\mathbf{z}_{k}^{H} \mathbf{M}^{-1} \mathbf{z}_{k}\right) \mathbf{z}_{k} \mathbf{z}_{k}^{H}
$$

where $\varphi$ is any real-valued weight function on $[0, \infty)$ that does not need to be related to the PDF of the underlying CES distribution. Thus $M$-estimators constitute a wide class of scatter matrix estimators. Existence and uniqueness of the solution of (2) as well as the convergence of the corresponding recursive algorithm have already been shown, provided the function $\varphi$ satisfies a set of general assumptions [11]. In particular, the resulting estimators are consistent estimators of the CM (up to a scale factor) [11].

For the particular function $\varphi(s)=\mathrm{m} / \mathrm{s}$, Tyler estimator $\widehat{\mathbf{M}}_{F P}$ is the solution (up to a scale factor) of the following equation

$$
\widehat{\mathbf{M}}_{F P}=\frac{m}{N} \sum_{k=1}^{N} \frac{\mathbf{z}_{k} \mathbf{z}_{k}^{H}}{\mathbf{z}_{k}^{H} \widehat{\mathbf{M}}_{F P}^{-1} \mathbf{z}_{k}}
$$

Moreover, for all $M$-estimators $\widehat{\mathbf{M}}$ verifying (2), one has

$$
\sqrt{N} \operatorname{vec}(\widehat{\mathbf{M}}-\mathbf{M}) \stackrel{d}{\rightarrow} \mathbb{C} N(\mathbf{0}, \boldsymbol{\Sigma}, \boldsymbol{\Omega})
$$

where $\boldsymbol{\Sigma}$ and $\boldsymbol{\Omega}$ are defined by

$$
\begin{array}{r}
\boldsymbol{\Sigma}=\nu_{1} \mathbf{M}^{T} \otimes \mathbf{M}+\nu_{2} \operatorname{vec}(\mathbf{M}) \operatorname{vec}(\mathbf{M})^{H}, \\
\boldsymbol{\Omega}=\nu_{1}\left(\mathbf{M}^{T} \otimes \mathbf{M}\right) \mathbf{K}+\nu_{2} \operatorname{vec}(\mathbf{M}) \operatorname{vec}(\mathbf{M})^{T}
\end{array}
$$

and where $\nu_{1}$ an $\nu_{2}$ are real scalars depending on the underlying CES distribution and the function $\varphi$ (see $[10,11]$ ). Note that the previous result is also valid for Tyler's estimator for any CES distribution with $\nu_{1}=(m+1) / m$ and $\nu_{2}=-(m+1) / m^{2}$ (see [13] for details).

\subsection{Wishart distribution}

The complex Wishart distribution $C W(N, \mathbf{M})$ is the distribution of $\sum_{k=1}^{N} \mathbf{x}_{k} \mathbf{x}_{k}^{H}$, when $\mathbf{x}_{k}$ are complex circular i.i.d. Gaussian vectors with zero mean and covariance matrix $\mathbf{M}$. Let

$$
\widehat{\mathbf{M}}_{S C M}=\frac{1}{N} \sum_{k=1}^{N} \mathbf{x}_{k} \mathbf{x}_{k}^{H}
$$

be the related SCM which will be also referred to as a Wishart matrix. Its asymptotic distribution is given by

$$
\sqrt{N} \operatorname{vec}\left(\widehat{\mathbf{M}}_{S C M}-\mathbf{M}\right) \stackrel{d}{\rightarrow} \mathbb{C} N\left(\mathbf{0}, \mathbf{M}^{T} \otimes \mathbf{M},\left(\mathbf{M}^{T} \otimes \mathbf{M}\right) \mathbf{K}\right) .
$$

Note that the previous result can be seen as a particular case of the result obtained for the $M$-estimators in (5) with $\nu_{1}=1$ and $\nu_{2}=0$ [15]. This shows that the $M$-estimators and Tyler estimator behave asymptotically as the SCM, the difference coming from the values of the quantities $\nu_{1}$ and $\nu_{2}$.

\subsection{ANMF test}

Consider the problem of detecting a known complex signal vector $\mathbf{p}$ from the received data $\mathbf{z}=\alpha \mathbf{p}+\mathbf{c}$, where $\mathbf{c}$ is the unobserved complex noise (clutter) random vector and $\alpha \in \mathbb{C}$ is a complex amplitude modeled as an unknown deterministic parameter or random parameter, depending on the application. In this paper, we assume that $\alpha$ is a complex unknown parameter accounting for both channel propagation effects and target backscattering and $\mathbf{p}$ is the transmitted known radar pulse vector. The problem of detecting the signal $\mathbf{p}$ can then be expressed as a the following binary hypothesis test

$$
\left\{\begin{array}{lll}
H_{0}: \mathbf{z}=\mathbf{c} & \mathbf{z}_{k}=\mathbf{c}_{k}, & k=1, \ldots, N \\
H_{1}: \mathbf{z}=\alpha \mathbf{p}+\mathbf{c} & \mathbf{z}_{k}=\mathbf{c}_{k}, & k=1, \ldots, N
\end{array}\right.
$$

where the $\mathbf{c}_{k} \sim \mathbb{C} E S_{m}\left(\boldsymbol{\mu}, \mathbf{M}, g_{\mathbf{c}}\right)$ are $N$ signal-free independent measurements, traditionally called the secondary data, 
used to estimate the background covariance matrix M. Assuming that the noise follows a CES distribution with covariance matrix $\mathbf{M}$, we investigate the properties of the following ANMF detector [1]

$$
H(\widehat{\mathbf{M}})=\frac{\left|\mathbf{p}^{H} \widehat{\mathbf{M}}^{-1} \mathbf{z}\right|^{2}}{\left(\mathbf{p}^{H} \widehat{\mathbf{M}}^{-1} \mathbf{p}\right)\left(\mathbf{z}^{H} \widehat{\mathbf{M}}^{-1} \mathbf{z}\right)} \underset{H_{1}}{\stackrel{H_{0}}{\lessgtr}} \lambda .
$$

Assuming that the SCM is used, the resulting PDF of $H\left(\widehat{\mathbf{M}}_{S C M}\right)$ is given by $f_{H\left(\widehat{\mathbf{M}}_{S C M}\right)}(u)=K(1-u)^{a-2}{ }_{2} F_{1}(a, a ; b ; u) \mathbf{1}_{[0, u]}(u)$ where $K=\frac{(a-1)(m-1)}{N+1}, a=N-m+2, b=N+2$ and ${ }_{2} F_{1}(\cdot)$ is the hypergeometric function [16]. The theoretical relationship between the detection threshold and the Probability of False Alarm (PFA) $P_{f a}=P\left(H(\widehat{\mathbf{M}})>\lambda \mid H_{0}\right)=$ $(1-\lambda)^{m-1}$ is defined as [17]

$$
P_{f a}=(1-\lambda)^{a-1}{ }_{2} F_{1}(a, a-1 ; b-1 ; \lambda) .
$$

Note that the distribution of $H(\widehat{\mathbf{M}})$ depends on the distribution of the vector $\mathbf{z}$. For any estimator $\widehat{\mathbf{M}}$ satisfying equation (2), one has the following result, conditionally to $\mathbf{z}$, derived in [14]

$$
\sqrt{N}(H(\widehat{\mathbf{M}})-H(\mathbf{M}))_{\mathbf{z}} \stackrel{d}{\rightarrow} N\left(0, \boldsymbol{\Sigma}_{H}\right)
$$

where $\boldsymbol{\Sigma}_{H}$ is defined by

$$
\boldsymbol{\Sigma}_{H}=2 \nu_{1} H(\mathbf{M})(H(\mathbf{M})-1)^{2} .
$$

Note that for Tyler estimator one has $\nu_{1}=(m+1) / m$.

\section{MAIN CONTRIBUTION}

This section is devoted to the main contribution of this paper. Let $\left(\mathbf{z}_{1}, \ldots, \mathbf{z}_{N}\right)$ be an $N$-sample of $m$-dimensional complex independent vectors with $\mathbf{z}_{k} \sim \mathbb{C} E S_{m}\left(\mathbf{0}, \mathbf{M}, g_{\mathbf{z}}\right), k=$ $1, \ldots, N$. These samples admit the following representation $\mathbf{z}_{k}=\sqrt{\tau_{k}} \mathbf{x}_{k} /\left\|\mathbf{x}_{k}\right\|$, where $\mathbf{x}_{k} \sim \mathbb{C} N_{m}(\mathbf{0}, \mathbf{M})$ and $\tau_{k}$ is an independant random variable whose p.d.f. is linked to the density generator $g_{\mathbf{z}}$ [11]. Note that Tyler estimator is distribution free, so it is identical when built either from the CES samples $\mathbf{z}_{k}$, or their Gaussian kernels $\mathbf{x}_{k}$ (unavailable in practice). Consider the SCM $\widehat{\mathbf{M}}_{S C M}$ built with $\left(\mathbf{x}_{1}, \ldots, \mathbf{x}_{N}\right)$ and the FP estimator $\widehat{\mathbf{M}}_{F P}$ built with $\left(\mathbf{z}_{1}, \ldots, \mathbf{z}_{N}\right)$. The following theorem shows that TyE-ANMF converges quickly towards the regime of the SCM-ANMF in a Gaussian context, even if the samples are CES distributed.

Theorem 3.1 Let us consider the ANMF test defined by (8). Thus, conditionally to the distributions of $z$, the asymptotic distribution of $H\left(\widehat{\boldsymbol{M}}_{F P}\right)-H\left(\widehat{\boldsymbol{M}}_{S C M}\right)$ is

$$
\sqrt{N}\left(H\left(\widehat{\boldsymbol{M}}_{F P}\right)-H\left(\widehat{\boldsymbol{M}}_{S C M}\right)\right) \stackrel{d}{\rightarrow} N\left(0, \boldsymbol{\Sigma}_{H}\right)
$$

where $\boldsymbol{\Sigma}_{T}$ is defined by

$$
\boldsymbol{\Sigma}_{T}=\frac{2}{m} H(\boldsymbol{M})(H(\boldsymbol{M})-1)^{2} .
$$

Before turning into the sketch of the proof, some comments are appropriate.

Remark 3.1 - The asymptotic variance in (13) is smaller than the ones of (11) when $\nu_{1}$ is greater than one 1. This result theoretically justifies that the behavior of $H\left(\widehat{\boldsymbol{M}}_{F P}\right)$ is closer to $H\left(\widehat{\boldsymbol{M}}_{S C M}\right)$ than to $H(\boldsymbol{M})$ $\left(\boldsymbol{\Sigma}_{T}<\boldsymbol{\Sigma}_{H}\right)$. An important consequence is a better detection performance prediction when using $H\left(\widehat{\boldsymbol{M}}_{S C M}\right)$ instead of $H(\boldsymbol{M})$.

- The asymptotic variance in (13) tends to 0 when the size $m$ increases: for high dimensional observations, this approximation is more accurate since $\boldsymbol{\Sigma}_{T} \ll \boldsymbol{\Sigma}_{H}$. Interestingly, this is in agreement with recent results obtained in [18] using large random matrix theory.

Proof 3.1 We provide here the main lines of the proof while the detailed one will be given in a forthcoming paper.

Let us first rewrite the right hand side of equation (12) as

$$
\begin{aligned}
& \sqrt{N}\left(H\left(\widehat{\boldsymbol{M}}_{F P}\right)-H\left(\widehat{\boldsymbol{M}}_{S C M}\right)\right) \\
& \left.=\sqrt{N}\left(H\left(\widehat{\boldsymbol{M}}_{F P}\right)-H(\boldsymbol{M})-H\left(\widehat{\boldsymbol{M}}_{S C M}\right)+H(\boldsymbol{M})\right)\right) \\
& =[1,-1]\left[\begin{array}{c}
\sqrt{N}\left(H\left(\widehat{\boldsymbol{M}}_{F P}\right)-H(\boldsymbol{M})\right) \\
\sqrt{N}\left(H\left(\widehat{\boldsymbol{M}}_{S C M}\right)-H(\boldsymbol{M})\right)
\end{array}\right]
\end{aligned}
$$

Therefore

$$
\begin{aligned}
\boldsymbol{\Sigma}_{H}^{(N)}= & N E\left[\left(H\left(\widehat{\boldsymbol{M}}_{F P}\right)-H\left(\widehat{\boldsymbol{M}}_{S C M}\right)\right)\right. \\
& \left.\left(H\left(\widehat{\boldsymbol{M}}_{F P}\right)-H\left(\widehat{\boldsymbol{M}}_{S C M}\right)\right)^{H}\right] \\
= & \boldsymbol{\Sigma}_{H 1}^{(N)}-2 \boldsymbol{\Sigma}_{H 2}^{(N)}+\boldsymbol{\Sigma}_{H 3}^{(N)}
\end{aligned}
$$

with

$\boldsymbol{\Sigma}_{H 1}^{(N)}=N E\left[\left(H\left(\widehat{\boldsymbol{M}}_{F P}\right)-H(\boldsymbol{M})\right)\left(H\left(\widehat{\boldsymbol{M}}_{F P}\right)-H(\boldsymbol{M})\right)^{H}\right]$

$\boldsymbol{\Sigma}_{H 2}^{(N)}=N E\left[\left(H\left(\widehat{\boldsymbol{M}}_{F P}\right)-H(\boldsymbol{M})\right)\left(H\left(\widehat{\boldsymbol{M}}_{S C M}\right)-H(\boldsymbol{M})\right)^{H}\right]$

$\boldsymbol{\Sigma}_{H 3}^{(N)}=N E\left[\left(H\left(\widehat{\boldsymbol{M}}_{S C M}\right)-H(\boldsymbol{M})\right)\left(H\left(\widehat{\boldsymbol{M}}_{S C M}\right)-H(\boldsymbol{M})\right)^{H}\right]$

As shown in [10], a first approximation of $H(\widehat{\boldsymbol{M}})$ yields

$$
H(\widehat{\boldsymbol{M}}) \simeq H(\boldsymbol{M})+H^{\prime}(\boldsymbol{M}) \operatorname{vec}(\widehat{\boldsymbol{M}}-\boldsymbol{M})
$$

which leads to $\boldsymbol{\Sigma}_{H 2}^{(N)} \simeq H^{\prime}(\boldsymbol{M}) \boldsymbol{\Sigma}_{M}^{(N)} H^{\prime}(\boldsymbol{M})^{H}$ where $\boldsymbol{\Sigma}_{M}^{(N)}=$ $E\left[\operatorname{vec}\left(\widehat{\boldsymbol{M}}_{F P}-\boldsymbol{M}\right) \operatorname{vec}\left(\widehat{\boldsymbol{M}}_{S C M}-\boldsymbol{M}\right)^{H}\right]$.

Let us now show that

$$
\boldsymbol{\Sigma}_{M}^{(N)} \rightarrow \boldsymbol{\Sigma}_{M}=\left(\boldsymbol{M}^{T} \otimes \boldsymbol{M}\right)-\frac{1}{m} \operatorname{vec}(\boldsymbol{M}) \operatorname{vec}(\boldsymbol{M})^{H} .
$$

Let us introduce the notations $\boldsymbol{\Delta}=\boldsymbol{M}^{-1 / 2} \widehat{\boldsymbol{M}} \boldsymbol{M}^{-1 / 2}-\boldsymbol{I}$ and $\boldsymbol{\delta}=\operatorname{vec}(\boldsymbol{\Delta})$ where $\widehat{\boldsymbol{M}}$ denotes an estimator of $\boldsymbol{M}$. Using these notations, $\boldsymbol{\Sigma}_{M}^{(N)}$ can be rewritten as

$$
\boldsymbol{\Sigma}_{M}^{(N)}=\left(\boldsymbol{M}^{T / 2} \otimes \boldsymbol{M}^{1 / 2}\right) N E\left[\boldsymbol{\delta}_{F P} \boldsymbol{\delta}_{S C M}^{H}\right]\left(\boldsymbol{M}^{T / 2} \otimes \boldsymbol{M}^{1 / 2}\right)^{H} .
$$

\footnotetext{
${ }^{1}$ which is the case for all the considered CM estimators.
} 
The next step is to derive the expression of $N E\left[\boldsymbol{\delta}_{F P} \boldsymbol{\delta}_{S C M}^{H}\right]$ when $N \rightarrow+\infty$. After few manipulations, one can show that

$$
N E\left[\boldsymbol{\delta}_{F P} \boldsymbol{\delta}_{S C M}^{H}\right] \rightarrow \frac{m+1}{m}\left[m \mathbf{A}-\operatorname{vec}(\boldsymbol{I}) \operatorname{vec}(\boldsymbol{I})^{T}\right]
$$

where $\mathbf{A}=E\left[\operatorname{vec}\left(\frac{\boldsymbol{x} \boldsymbol{x}^{H}}{\boldsymbol{x}^{H} \boldsymbol{x}}\right) \operatorname{vec}\left(\boldsymbol{x} \boldsymbol{x}^{H}\right)^{H}\right]$, with $\boldsymbol{x} \sim \mathbb{C} N(\mathbf{0}, \boldsymbol{M})$. Finally, a computation of A leads to (17).

Using $H^{\prime}(\boldsymbol{M}) \operatorname{vec}(\boldsymbol{M})=0$ (see [10]), one has

$$
\boldsymbol{\Sigma}_{H 2}^{(N)} \rightarrow \boldsymbol{\Sigma}_{H 2}=H^{\prime}(\boldsymbol{M})\left(\boldsymbol{M}^{T} \otimes \boldsymbol{M}\right) H^{\prime}(\boldsymbol{M})^{H}
$$

Using the following expression of $H^{\prime}(M)$ derived in [14]

$$
\begin{aligned}
H^{\prime}(\boldsymbol{M})= & -H(\boldsymbol{M})\left[\frac{\operatorname{vec}\left(\boldsymbol{p} \boldsymbol{z}^{H}\right)}{\left(\boldsymbol{p}^{H} \boldsymbol{M}^{-1} \boldsymbol{z}\right)}+\frac{v e c^{H}\left(z \boldsymbol{p}^{H}\right)}{\left(\boldsymbol{z}^{H} \boldsymbol{M}^{-1} \boldsymbol{p}\right)}\right. \\
& \left.-\frac{v e c^{H}\left(\boldsymbol{p} \boldsymbol{p}^{H}\right)}{\left(\boldsymbol{p}^{H} \boldsymbol{M}^{-1} \boldsymbol{p}\right)}-\frac{v e c\left(z z^{H}\right)}{\left(\boldsymbol{z}^{H} \boldsymbol{M}^{-1} \boldsymbol{z}\right)}\right]\left(\boldsymbol{M}^{T} \otimes \boldsymbol{M}\right)^{-1},
\end{aligned}
$$

then, one obtains the following final result

$$
\begin{aligned}
\boldsymbol{\Sigma}_{H} & =2\left(\frac{m+1}{m}-2+1\right) H(\boldsymbol{M})(H(\boldsymbol{M})-1)^{2} \\
& =\frac{2}{m} H(\boldsymbol{M})(H(\boldsymbol{M})-1)^{2} .
\end{aligned}
$$

This concludes the proof.

\section{SIMULATION RESULTS}

This section considers the ANMF built with the FP and SCM estimators. The results are obtained for complex Gaussiandistributed $^{2}$ zero-mean data with covariance matrix $\mathbf{M}$ whose elements are defined by $M_{i, j}=\rho^{|i-j|}, \quad i=1, \ldots, m$. The correlation coefficient $\rho$ is set to 0.5. The operator $\overline{\mathbf{A}}$ is defined as the empirical mean of the quantities $\mathbf{A}(i)=\operatorname{var}(H(\widehat{\mathbf{M}}(i)))$ computed using $I$ Monte Carlo runs.

Figure 1 illustrates the theoretical result of Theorem 3.1. The empirical variance of the difference between the TyEANMF and the SCM-ANMF is compared to the theoretical result. One can see that the error decreases very fast as the number $N$ of samples increases. Furthermore, simulations show that the approximation is also valid for small $N$.

Figure 2 displays the empirical variances of the TyEANMF and of the difference between TyE-ANMF and SCMANMF (Theorem 3.1). It also displays the theoretical result that has been derived in (13) (blue horizontal line). First, one can note that the variances of the TyE-ANMF largely exceed the variance of the difference, which supports the idea of approximating the properties of TyE-ANMF with the well-known ones of the SCM-ANMF in a Gaussian context. Second, one can observe that the empirical results tend to the theoretical asymptotic values, which is in agreement with our statement.

\footnotetext{
${ }^{2}$ Note that a change in the underlying distribution will not affect the results since Tyler estimator is distribution-free over the class of CES.
}

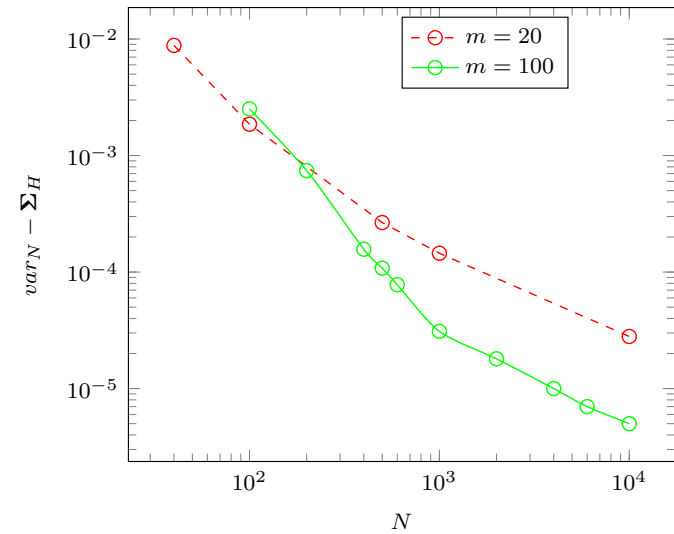

Fig. 1. Difference between the empirical variance of the difference of two ANMF tests $\left(\operatorname{var}_{N}=\operatorname{var}\left(\sqrt{N}\left(H\left(\widehat{\mathbf{M}}_{F P}\right)-\right.\right.\right.$ $\left.\left.H\left(\widehat{\mathbf{M}}_{S C M}\right)\right)\right)$ ) and the theoretical result (Eq. (13)) versus the number $N$ of observations for $m=20$ and $m=100$.

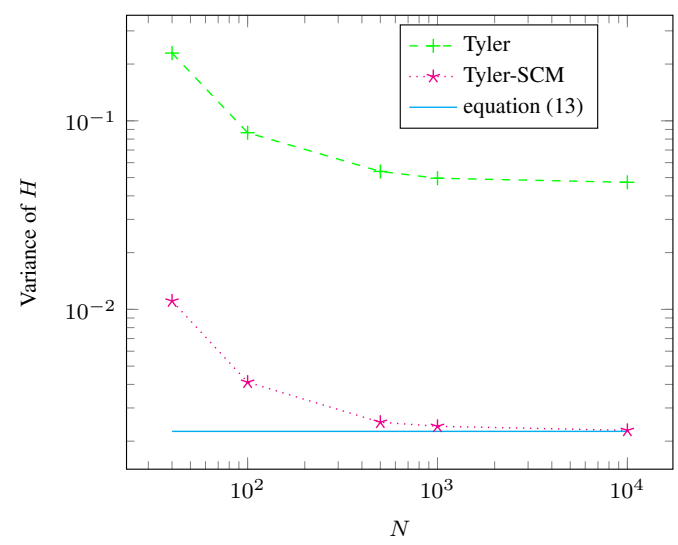

Fig. 2. Empirical variance of the TyE-ANMF, the SCMANMF (obtained from (10)) and of the difference between them and the theoretical result (equation (13)) for $m=20$.

\section{CONCLUSION}

This paper analyzed the asymptotic distribution of the ANMF built with Tyler estimator (TyE-ANMF) when the observed data have a CES distribution. We have shown that the TyEANMF behaves as the SCM-ANMF in a Gaussian context, even if the samples have a CES distribution. This is important in practice since it allows one to use the TyE-ANMF for detection purposes and to theoretically adjust parameters (e.g., the detection threshold) thanks to the SCM-ANMF properties. Here, the SCM plays only a theoretical role since for CESdistributed data, it is neither robust nor accurate. In general, this result supports the idea of approximating the properties of the Tyler estimator by the ones of Wishart distributions. Since the Wishart distribution is well-known, this allows us to better understand the behavior of Tyler estimator in the context of CES-distributed data. 


\section{REFERENCES}

[1] E. Conte, M. Lops, and G. Ricci, "Asymptotically optimum radar detection in compound-Gaussian clutter," IEEE Transactions on Aerospace and Electronic Systems, vol. 31, no. 2, pp. 617-625, April 1995.

[2] F. Gini, "Sub-optimum coherent radar detection in a mixture of K-distributed and Gaussian clutter," IEEE Proceedings - Radar, Sonar and Navigation, vol. 144, no. 1, pp. 39-48, February 1997.

[3] S. Kraut and L. L. Scharf, "The CFAR adaptive subspace detector is a scale-invariant GLRT," IEEE Transactions on Signal Processing, vol. 47, no. 9, pp. 25382541, September 1999.

[4] S. Kraut, L. L. Scharf, and R. W. Butler, "The adaptive coherence estimator: a uniformly most-powerfulinvariant adaptive detection statistic," IEEE Transactions on Signal Processing, vol. 53, no. 2, pp. 427-438, February 2005.

[5] S. Kraut, L. L. Scharf, and L. T. McWhorter, "Adaptive subspace detectors," IEEE Transactions on Signal Processing, vol. 49, no. 1, pp. 1-16, January 2001.

[6] E. Conte, A. De Maio, and G. Ricci, "Recursive estimation of the covariance matrix of a compound-Gaussian process and its application to adaptive CFAR detection," IEEE Transactions on Signal Processing, vol. 50, no. 8, pp. 1908-1915, August 2002.

[7] M. Bilodeau and D. Brenner, Theory of Multivariate Statistics, New York, NY. USA:Springer-Verlag, 1999.

[8] P. J. Huber, "Robust estimation of a location parameter," The Annals of Mathematical Statistics, vol. 35, no. 1, pp. 73-101, January 1964.

[9] R. A. Maronna, "Robust $M$-estimators of multivariate location and scatter," Annals of Statistics, vol. 4, no. 1, pp. 51-67, January 1976.

[10] M. Mahot, F. Pascal, P. Forster, and J.-P. Ovarlez, "Asymptotic properties of robust complex covariance matrix estimates," IEEE Transactions on Signal Processing, vol. 61, no. 13, pp. 3348-3356, July 2013.

[11] E. Ollila, D.E. Tyler, V. Koivunen, and H.V. Poor, "Complex elliptically symmetric distributions: Survey, new results and applications," IEEE Transactions on Signal Processing, vol. 60, no. 11, pp. 5597-5625, November 2012.

[12] F. Pascal, Y. Chitour, J.-P. Ovarlez, P. Forster, and P. Larzabal, "Covariance structure maximum-likelihood estimates in compound Gaussian noise: existence and algorithm analysis," IEEE Transactions on Signal Processing, vol. 56, no. 1, pp. 34-48, January 2008.

[13] F. Pascal, P. Forster, J.-P. Ovarlez, and P. Larzabal, "Performance analysis of covariance matrix estimates in impulsive noise," IEEE Transactions on Signal Processing, vol. 56, no. 6, pp. 2206-2217, June 2008.

[14] F. Pascal and J.-P. Ovarlez, "Asymptotic properties of the robust ANMF," in Proc. ICASSP, Brisbane, Australia, April 2015.

[15] T.W. Anderson, An Introduction to Multivariate Statistical Analysis, Wiley Series in Probability and Statistics. Wiley, 2003.

[16] Milton Abramowitz and Irene A Stegun, Handbook of mathematical functions: with formulas, graphs, and mathematical tables, vol. 55, Courier Corporation, 1964.

[17] F. Pascal, J. P. Ovarlez, P. Forster, and P. Larzabal, "Constant false alarm rate detection in spherically invariant random processes," pp. 2143-2146, Sept 2004.

[18] R. Couillet, F. Pascal, and J. W. Silverstein, "The Random Matrix Regime of Maronna's $M$-estimator with elliptically distributed samples," Journal of Multivariate Analysis, vol. 139, pp. 56-78, July 2015. arXiv:1311.7034. 